\title{
Aplicação de Modelos de Regressão e de Adaptabilidade e Estabilidade na Identificação de Cultivares de Aveia Branca com Maior Resistência Genética a Doenças Foliares
}

\author{
Odenis Alessi ${ }^{1}$ \\ Eldair Fabricio Dornelles ${ }^{2}$ \\ Ângela Teresinha Woschinski de Mamann ${ }^{3}$ \\ Adriana Roselia Kraisig ${ }^{4}$ \\ Luana Henrichsen ${ }^{5}$ \\ Anderson Marolli ${ }^{6}$ \\ Vanessa Pansera ${ }^{7}$ \\ José Antonio Gonzalez da Silva ${ }^{8}$ \\ Departamento de Ciências Exatas e Engenharias, DECEng/UNIJUÍ, Ijuí, RS
}

\begin{abstract}
Resumo. Modelos de regressão e o modelo de adaptabilidade e estabilidade de Eberhart \& Russel podem contribuir no melhoramento de manejos de diferentes cultivares. Na aveia, podem auxiliar na identificação de cultivares mais resistentes às doenças foliares. Com isso, pode-se evitar o uso indiscriminado de fungicida e diminuir os riscos de contaminação do grão e do meio ambiente. Desta forma, o objetivo deste trabalho é empregar os modelos de regressão e o modelo de adaptabilidade e estabilidade para identificação das melhores cultivares de aveia recomendadas para cultivo no Brasil. O delineamento experimental foi de blocos casualizados seguindo um esquema fatorial $22 \times 4$ para 22 cultivares de aveia branca e 4 condições de aplicação de fungicida. Após a coleta dos dados experimentais e aplicação dos modelos de regressão e de adaptabilidade e estabilidade, conclui-se que a cultivar URS Altiva apresenta melhores resultados em promover maior produtividade de grãos com possibilidade de menor uso de fungicida.
\end{abstract}

Palavras-chave. Análise de Regressão, Adaptabilidade e Estabilidade, Resistência Genética

\section{Introdução}

A utilização da análise de regressão e do modelo de adaptabilidade e estabilidade de Eberhart \& Russel vem possibilitando a identificação de manejos mais sustentáveis e o

\footnotetext{
${ }^{1}$ Bolsista CAPES/BRASIL. odenisalessi@hotmail.com

${ }^{2}$ eldair.dornelles@gmail.com

3 angelademamann@hotmail.com

${ }^{4}$ maryshelei@yahoo.com.br

${ }^{5}$ luanabehnenh@gmail.com

${ }^{6}$ marollia@yahoo.com.br

7vpansera@hotmail.com

8jagsfaem@yahoo.com.br
} 
conhecimento fisiológico de diferentes culturas na agricultura $[2,8]$. O uso de análises por modelos de regressão permite estabelecer relações entre uma variável dependente e uma ou mais variáveis independentes [5]. Já o método proposto por Eberhart \& Russel (1966) para o modelo de adaptabilidade e estabilidade mede a resposta de diferentes genótipos frente às variações ambientais. Os autores estabeleceram neste método um parâmetro para o cálculo da adaptabilidade de um genótipo e outro para estimar a estabilidade [4].

O cultivo da aveia branca ocorre de forma expressiva no sul do Brasil [7]. Referente ao seu cultivo, a aplicação de fungicida pode ocorrer de maneira frequente, visto que a mesma é suscetível ao ataque de doenças foliares [3]. Buscando evitar o uso excessivo destes insumos agrícolas, busca-se identificar cultivares que apresentem maior resistência genética a estas doenças. Com isso, pode-se evitar a contaminação dos grãos e do meio ambiente pelo uso indiscriminado de fungicida [2].

Nesta perspectiva, o uso de modelos de regressão pode identificar a relação entre a produtividade de grãos e o número de aplicações de fungicida evidenciando os melhores desempenhos entre as cultivares. Já o modelo de adaptabilidade e estabilidade de Eberhart \& Russel pode ser importante na identificação de cultivares de aveia mais responsivas às aplicações de fungicida e que, consequentemente, sejam mais resistentes às doenças foliares, possibilitando a redução do número de aplicações do mesmo $[1,3]$. Desta forma, o objetivo deste trabalho é empregar a análise de regressão e o modelo de adaptabilidade e estabilidade de Eberhart \& Russel na identificação de cultivares de aveia com maior resistência genética às doenças foliares e estáveis à expressão da produtividade de grãos em função do momento e número de aplicações do fungicida, considerando um ano agrícola intermediário para a produtividade de grãos e favorável ao progresso das doenças foliares.

\section{Metodologia}

O trabalho foi desenvolvido no ano agrícola de 2015 na área experimental do Instituto Regional de Desenvolvimento Rural (IRDeR) pertencente ao Departamento de Estudos Agrários (DEAg) da UNIJUÍ. O delineamento experimental foi de blocos casualizados seguindo um esquema fatorial $22 \times 4$, para 22 cultivares de aveia branca e 4 condições de aplicações de fungicida. As condições adotadas nas aplicações de fungicida seguiram a seguinte forma: sem aplicação de fungicida, uma aplicação aos 60 Dias Após a Emergência (DAE), duas aplicações (uma aos 60 e a outra aos 75 DAE) e com três aplicações (uma aos 60, outra aos 75 e a última aos $90 \mathrm{DAE}$ ). A avaliação da área foliar ocorreu com a coleta de três folhas superiores de três plantas de cada parcela aos 105 DAE. As mesmas foram digitalizadas utilizando o leitor de área foliar e o software WinDIAS (Copyright 2012 Delta-T Devices Limited). Ao final do ciclo, realizou-se o corte das três linhas centrais de cada parcela para a obtenção da produtividade. Após a obtenção dos dados foram geradas as médias das cultivares quanto à produtividade de grãos e área foliar necrosada. Primeiramente, aplicou-se a análise de variância para detecção dos efeitos principais e de interação e, em seguida, realizou-se análise de médias onde classificou-se as cultivares em: superior $\left(^{S}\right)$, mediana e inferior $\left({ }^{I}\right)$. O critério utilizado na classificação das cultivares corresponde a média mais ou menos um desvio padrão. Para a estimação da produtividade 
de grãos em função do número de aplicações de fungicida, os dados foram submetidos a uma análise de regressão linear simples $\left(Y=b_{0} \pm b_{1} x\right)$. Quanto à identificação das cultivares com capacidade de adaptabilidade frente às alterações climáticas locais, responsivas ao uso de fungicida e estáveis a produtividade de grãos, os dados mensurados foram submetidos ao modelo de adaptabilidade e estabilidade de Eberhart \& Russell (1966), dado pela equação $(1)$ :

$$
Y_{i j}=b_{0 i}+b_{1 i} I_{j}+S_{i j}^{2}+\bar{\epsilon}_{i j}
$$

onde: $Y_{i j}$ é a média do genótipo $i$ no ambiente $j, b_{0 i}$ é a média geral no genótipo $i, b_{1 i}$ é o coeficiente de regressão linear que mede a resposta do i-ésimo genótipo à variação do ambiente, $I_{j}$ é o índice ambiental padronizado, $S_{i j}^{2}$ são os desvios de regressão linear e $\bar{\epsilon}_{i j}$ é o erro experimental médio. Este modelo foi escolhido pois permite a codificação de um índice ambiental no modelo de regressão linear e possibilita estimar simultaneamente os parâmetros de adaptabilidade e estabilidade das cultivares utilizadas. Para classificação das cultivares quanto à produtividade de grãos e área foliar necrosada, realizou-se o agrupamento de médias por Scott \& Knott [9]. As análises estatísticas foram realizadas com o auxílio do software livre GENES.

\section{Resultados e Discussão}

Na Figura 1, observa-se os dados meteorológicos referentes ao ano agrícola de 2015 e aos momentos de aplicação do fungicida, sendo estes obtidos de uma estação meteorológica localizada no IRDeR/DEag/UNIJUÍ.

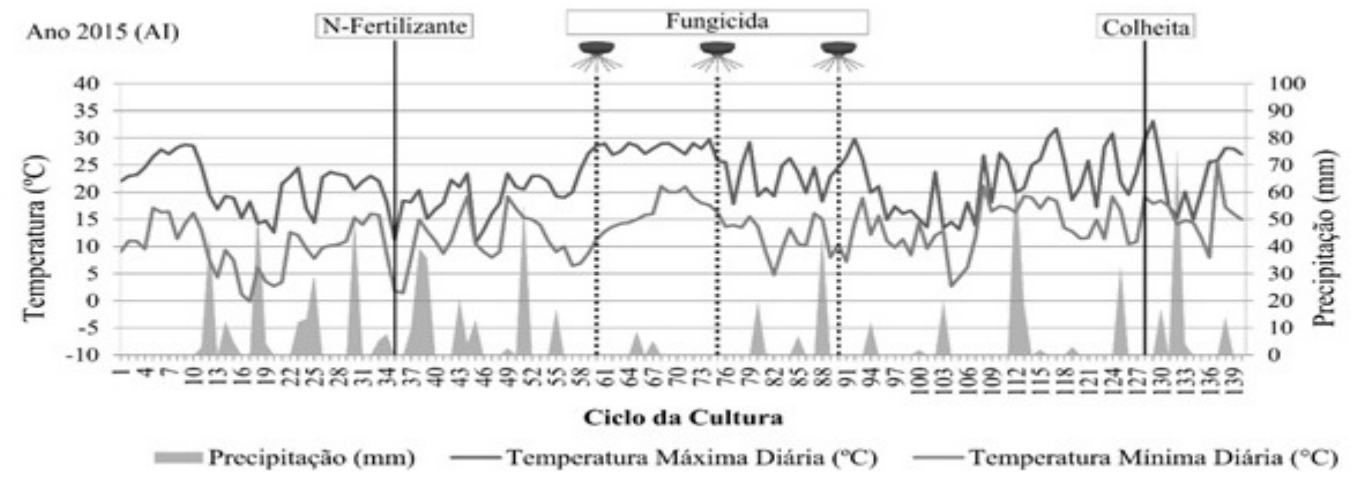

Figura 1: Dados meteorológicos e ciclo da cultura da aveia.

As precipitações pluviométricas ocorreram de forma similar à média histórica dos últimos 25 anos durante o ciclo da cultura, ocorrendo um maior volume de chuvas durante a fase vegetativa. Pelas informações de temperatura, de precipitação durante o ciclo de cultivo da aveia e da produtividade de grãos obtida, os autores do estudo a partir de suas expertises, classificaram o ano de 2015 como intermediário (AI) ao cultivo e favorável ao desenvolvimento de doenças foliares. 
Na análise de médias (Tabela 1) as cultivares URS Altiva e URS Guria apresentaram os melhores desempenhos quanto à produtividade de grãos. Para estas, as duas aplicações de fungicida foram suficientes para atingirem os valores mais expressivos de produtividade, similar a média geral obtida com três aplicações para as 22 cultivares. Além disto, a cultivar URS Altiva, assim como a URS Brava, apresentou forte incremento de produtividade com a terceira aplicação. Quanto à área foliar necrosada, as cultivares citadas tiveram suas áreas foliares comprometidas de forma mediana nas quatro condições de uso de fungicida aplicadas.

Tabela 1: Média de produtividade de grãos e área foliar necrosada em cultivares de aveia nas condições de uso de fungicida.

\begin{tabular}{|c|c|c|c|c|c|c|c|c|}
\hline \multirow[t]{2}{*}{ Cultivar } & \multicolumn{4}{|c|}{ Fungicida } & \multicolumn{4}{|c|}{ Fungicida } \\
\hline & $\mathrm{SF}$ & $\begin{array}{l}\mathrm{CF}_{1} \\
(60)\end{array}$ & $\begin{array}{c}\mathrm{CF}_{2} \\
(60 / 75)\end{array}$ & $\begin{array}{c}\mathrm{CF}_{3} \\
(60 / 75 / 90)\end{array}$ & $\mathrm{SF}$ & $\begin{array}{l}\mathrm{CF}_{1} \\
(60)\end{array}$ & $\begin{array}{c}\mathrm{CF}_{2} \\
(60 / 75)\end{array}$ & $\begin{array}{c}\mathrm{CF}_{3} \\
(60 / 75 / 90)\end{array}$ \\
\hline & \multicolumn{4}{|c|}{ PG $\left(\mathrm{kg} \mathrm{ha}^{-1}\right)$} & \multicolumn{4}{|c|}{$\mathrm{AFN}_{105 D A E}(\%)$} \\
\hline URS Altiva & $2321^{S}$ & $2696^{S}$ & $3487^{S}$ & $4236^{S}$ & 69 & 30 & 7 & 2 \\
\hline URS Brava & 1229 & 2393 & 3191 & $3993^{S}$ & 53 & 14 & 7 & 2 \\
\hline URS Guará & 1486 & 2083 & 3166 & 3453 & 77 & 20 & 9 & $0^{S}$ \\
\hline URS Estampa & 1555 & 2393 & 2822 & 3302 & $28^{S}$ & 22 & $19^{I}$ & 10 \\
\hline URS Corona & 804 & 2030 & 2824 & 3333 & $98^{I}$ & $90^{I}$ & 6 & $22^{I}$ \\
\hline URS Torena & 1348 & 2377 & 2783 & $2892^{I}$ & 69 & 41 & $18^{I}$ & 16 \\
\hline URS Charrua & 1616 & $2662^{S}$ & 3078 & 3107 & 63 & 24 & 10 & 4 \\
\hline URS Guria & $1949^{S}$ & $2681^{S}$ & $3413^{S}$ & 3517 & 62 & 16 & 8 & 2 \\
\hline URS Tarimba & 1386 & $1646^{I}$ & $2672^{I}$ & 3160 & 55 & 28 & 14 & 11 \\
\hline URS Taura & 863 & 1813 & $2152^{I}$ & $2775^{I}$ & 89 & 26 & 14 & 9 \\
\hline URS 21 & 1552 & 2192 & 3071 & 3104 & 68 & 26 & 14 & 9 \\
\hline FAEM 007 & $579^{I}$ & $1194^{I}$ & 2922 & $2923^{I}$ & 85 & $69^{I}$ & 10 & $24^{I}$ \\
\hline FAEM 006 & $749^{I}$ & 2035 & 3265 & 3660 & $97^{I}$ & 56 & $4^{S}$ & 14 \\
\hline FAEM 5 Chiarasul & $727^{I}$ & $1304^{I}$ & 3353 & $3923^{S}$ & $96^{I}$ & $60^{I}$ & 6 & 11 \\
\hline FAEM 4 Carlasul & 1352 & 2165 & $3414^{S}$ & 3514 & 75 & 33 & 9 & 16 \\
\hline Brisasul & 1066 & 2237 & $3516^{S}$ & 3700 & $37^{\mathrm{S}}$ & 34 & 9 & 10 \\
\hline Barbarasul & $680^{I}$ & 1854 & $3579^{S}$ & 3739 & 86 & $82^{I}$ & 12 & 4 \\
\hline URS Fapa Slava & 1189 & 1719 & 3096 & 3159 & 49 & 46 & $24^{I}$ & $17^{I}$ \\
\hline IPR Afrodite & $566^{I}$ & $1527^{I}$ & 2802 & 3382 & $94^{I}$ & 37 & 5 & 4 \\
\hline UPFPS Farroupilha & 1324 & 2448 & 3215 & 3464 & 61 & 28 & $4^{S}$ & 2 \\
\hline UPFA Ouro & 1386 & 2290 & 2791 & 3378 & $31^{S}$ & $6^{S}$ & 5 & $0^{S}$ \\
\hline UPFA Gaudéria & 1308 & 2163 & $2601^{I}$ & 3222 & $27^{S}$ & 15 & 12 & 8 \\
\hline Média Geral & 1229 & 2086 & 3055 & 3406 & 67 & 36 & 10 & 9 \\
\hline DP & 448 & 422 & 353 & 368 & 23 & 22 & 5 & 7 \\
\hline$\overline{\text { Superior }\left({ }^{S}\right)}$ & 1677 & 2508 & 3409 & 3774 & 44 & 14 & 5 & 2 \\
\hline Inferior $\left({ }^{I}\right)$ & 781 & 1665 & 2702 & 3038 & 89 & 59 & 15 & 16 \\
\hline \multicolumn{9}{|c|}{$\begin{array}{l}\mathrm{SF}=\text { sem fungicida; } \mathrm{CF}_{1}=\text { uma aplicação de fungicida; } \mathrm{CF}_{2}=\text { duas aplicações de fungicida; } \mathrm{CF}_{3}=\text { três aplicações } \\
\text { de fungicida; }(60)=\text { dia da aplicaçâo do fungicida após a emergência; }(60 / 75)=\text { dias da primeira e segunda } \\
\text { aplicação de fungicida após a emergência, respectivamente; }(60 / 75 / 90)=\text { dias da primeira, segunda e terceira } \\
\text { aplicação de fungicida após a emergência, respectivamente; } \mathrm{PG}=\text { produtividade de grãos; } A F N_{105 D A E}=\text { área } \\
\text { foliar necrosada avaliada aos } 105 \text { dias após a emergência; } S=\text { superior à média mais um desvio padrão para a } \\
\text { variável PG e inferior à média menos um desvio padrão para a variável } A F N_{105 D A E} ;=\text { inferior à média menos } \\
\text { um desvio padrão para a variável } \mathrm{PG} \text { e superior à média mais um desvio padrão para a variável } A F N_{105 D A E} ; \\
\mathrm{DP}=\text { desvio padrão. }\end{array}$} \\
\hline
\end{tabular}


Na Tabela 2, de regressão linear da produtividade de grãos e área foliar necrosada, observa-se que a cultivar URS Altiva foi a que apresentou melhor desempenho quanto à produtividade de grãos entre as cultivares. Em relação à área foliar necrosada, seu desempenho frente ao ataque das doenças foi mediano. É valido salientar que pode-se perceber através da utilização das regressões, o incremento da produtividade de grãos e o decremento da área foliar necrosada em função do número de aplicações de fungicida.

Tabela 2: Regressão linear da produtividade de grãos e área foliar necrosada em cultivares de aveia em função do número de aplicações de fungicida.

\begin{tabular}{|c|c|c|c|c|c|c|}
\hline \multirow[t]{2}{*}{ Cultivar } & \multicolumn{3}{|c|}{ Produtividade de grãos $\left(\mathrm{kg} h a^{-1}\right)$} & \multicolumn{3}{|c|}{$A F N_{105 D A E}(\%)$} \\
\hline & $b_{0} \pm b_{1} x$ & $R^{2}$ & $P\left(b_{1} x\right)$ & $b_{0} \pm b_{1} x$ & $R^{2}$ & $P\left(b_{1} x\right)$ \\
\hline URS Altiva & ${ }^{S} 2204+654 x$ & 98 & * & $60-22,3 x$ & 89 & $*$ \\
\hline URS Brava & $1338+908 x$ & 99 & $*$ & $43-16 x$ & 79 & $*$ \\
\hline URS Guará & $1500+698 x$ & 96 & $*$ & $62-23,9 x$ & 81 & $*$ \\
\hline URS Estampa & $1667+567 x$ & 97 & $*$ & $S_{28}-{ }^{S} 5,7 x$ & 97 & * \\
\hline URS Corona & $990+838 x$ & 96 & $*$ & ${ }^{I} 100-{ }^{I} 31,0 x$ & 74 & $*$ \\
\hline URS Torena & $1594+{ }^{I} 503 x$ & 84 & $*$ & $63-18,2 x$ & 90 & $*$ \\
\hline URS Charrua & ${ }^{S} 1882+{ }^{I} 489 x$ & 82 & $*$ & $54-19,2 x$ & 86 & $*$ \\
\hline URS Guria & ${ }^{S} 2075+{ }^{I} 543 x$ & 93 & $*$ & $50-18,8 x$ & 79 & $*$ \\
\hline URS Tarimba & $1264+635 x$ & 95 & $*$ & $49-14,9 x$ & 88 & $*$ \\
\hline URS Taura & $990+607 x$ & 96 & $*$ & $72-25,1 x$ & 77 & $*$ \\
\hline URS 21 & $1650+{ }^{I} 553 x$ & 91 & $*$ & $58-19,1 x$ & 84 & $*$ \\
\hline FAEM 007 & ${ }^{I} 591+876 x$ & 89 & $*$ & ${ }^{I} 83-24,0 x$ & 77 & $*$ \\
\hline FAEM 006 & $933+{ }^{S} 996 x$ & 96 & $*$ & ${ }^{I} 88-{ }^{I} 30,1 x$ & 83 & $*$ \\
\hline FAEM 5 Chiarasul & ${ }^{I} 581+{ }^{S} 1164 x$ & 93 & $*$ & ${ }^{I} 90-{ }^{I} 30,8 x$ & 87 & $*$ \\
\hline FAEM 4 Carlasul & $1451+774 x$ & 92 & $*$ & $63-20,2 x$ & 77 & $*$ \\
\hline Brisasul & $1253+918 x$ & 93 & $*$ & $38-{ }^{S} 10,5 x$ & 83 & $*$ \\
\hline Barbarasul & ${ }^{I} 828+{ }^{S} 1090 x$ & 93 & $*$ & ${ }^{I} 94-{ }^{I} 31,8 x$ & 86 & * \\
\hline URS Fapa Slava & $1197+729 x$ & 90 & $*$ & $52-11,8 x$ & 92 & $*$ \\
\hline IPR Afrodite & ${ }^{I} 611+{ }^{S} 972 x$ & 98 & $*$ & $80-{ }^{I} 30,2 x$ & 85 & $*$ \\
\hline UPFPS Farroupilha & $1535+719 x$ & 93 & $*$ & $54-20,1 x$ & 89 & * \\
\hline UPFA Ouro & $1490+648 x$ & 98 & $*$ & ${ }^{S} 25-{ }^{S} 9,3 x$ & 76 & $*$ \\
\hline UPFA Gaudéria & $1396+618 x$ & 98 & $*$ & ${ }^{S} 24-{ }^{S} 5,8 x$ & 91 & $*$ \\
\hline$\overline{\text { Geral }}$ & $1319+750 x$ & & & $60-19,9 x$ & & \\
\hline DP & $453+194 x$ & & & $22-8,1 x$ & & \\
\hline Superior & $1722+944 x$ & & & $38-11,8 x$ & & \\
\hline Inferior & $866+555 x$ & & & $82-28,0 x$ & & \\
\hline
\end{tabular}

$A F \overline{N_{105} D A E}=$ área foliar necrosada avaliada aos 105 dias após a emergência; $P\left(b_{1} x\right)=$ parâmetro que mede a inclinação da reta pela probabilidade de T a $5 \%$ de erro; $R^{2}=$ coeficiente de determinação; $*=$ significativo a $5 \%$ de probabilidade de erro; ${ }^{S}=$ valor superior à média mais um desvio padrão quando relacionado à produtividade de grãos ou valor inferior à média menos um desvio padrão quando relacionado à área foliar necrosada; ${ }^{I}=$ valor inferior à média menos um desvio padrão quando relacionado à produtividade de grãos ou valor superior à média mais um desvio padrão quando relacionado à área foliar necrosada; $\mathrm{DP}=$ desvio padrão.

$\mathrm{Na}$ análise da adaptabilidade e estabilidade (Tabela 3), as maiores produtividades de grãos foram apresentadas pelas cultivares URS Altiva e URS Guria. Além disso, estas cultivares apresentaram adaptabilidade específica a ambientes desfavoráveis $\left(b_{1}<1\right)$, ou seja, elas apresentam potencial de produtividade mesmo em condições restritivas ocasionadas pela redução do número de aplicações de fungicida. Entretanto, a cultivar URS 
Altiva evidenciou instabilidade sobre a produtividade pelo número de aplicações, o que não ocorre na cultivar URS Guria, que apresentou estabilidade. Ainda pode-se observar que as cultivares URS Guará, FAEM 4 Carlasul e UPFPS Farroupilha evidenciaram desempenho intermediário de produtividade, mostrando adaptabilidade geral $\left(b_{1}=1\right)$ com estabilidade. Em relação à área foliar necrosada, das citadas, apenas as cultivares URS Altiva e UPFPS Farroupilha evidenciaram as menores médias com adaptabilidade geral e estabilidade. Destaca-se que a adaptabilidade geral relacionada à produtividade de grãos denota o desempenho de cultivares que respondem bem ao estímulo ambiental independente da condição favorável ou desfavorável pelo uso de fungicida.

Tabela 3: Parâmetros de adaptabilidade e estabilidade em cultivares de aveia em função do uso de fungicida sobre a produtividade de grãos e área foliar necrosada.

\begin{tabular}{|c|c|c|c|c|c|c|c|c|}
\hline \multirow[t]{2}{*}{ Cultivar } & \multicolumn{4}{|c|}{$\mathrm{PG}\left(\mathrm{kg} h a^{-1}\right)$} & \multicolumn{4}{|c|}{$A F N_{105 D A E}(\%)$} \\
\hline & $b_{0}$ & $b_{1}$ & $S^{2}$ & $R^{2}$ & $b_{0}$ & $b_{1}$ & $S^{2}$ & $R^{2}$ \\
\hline$\overline{\text { URS Altiva }}$ & $3185 \mathrm{a}$ & $0,83^{*}$ & $69778^{*}$ & 92 & $26,8 \mathrm{c}$ & $1,12^{\text {ns }}$ & $-11,43^{\text {ns }}$ & $\overline{99}$ \\
\hline URS Brava & $2702 \mathrm{c}$ & $1,19^{*}$ & $34084^{*}$ & 98 & $19,2 \mathrm{c}$ & $0,82^{*}$ & $46,88^{*}$ & 91 \\
\hline URS Guará & $2547 \mathrm{c}$ & $0,93^{n s}$ & $228^{n s}$ & 99 & $26,4 \mathrm{c}$ & $1,21^{*}$ & $121,12^{*}$ & 92 \\
\hline URS Estampa & $2518 \mathrm{c}$ & $0,74^{*}$ & $13258^{\text {ns }}$ & 97 & $19,7 \mathrm{c}$ & $0,24^{*}$ & $-7,15^{n s}$ & 80 \\
\hline URS Corona & $2248 \mathrm{~d}$ & $1,11^{*}$ & $13706^{n s}$ & 99 & $54,2 \mathrm{a}$ & $1,55^{*}$ & $547,56^{*}$ & 82 \\
\hline URS Torena & $2350 \mathrm{~d}$ & $0,69^{*}$ & $46066^{*}$ & 92 & $36,0 \mathrm{~b}$ & $0,91^{\text {ns }}$ & $-23,52^{n s}$ & 100 \\
\hline URS Charrua & $2616 \mathrm{c}$ & $0,67^{*}$ & $59209^{*}$ & 90 & $25,1 \mathrm{c}$ & $0,96^{n s}$ & $18,43^{n s}$ & 96 \\
\hline URS Guria & $2890 \mathrm{~b}$ & $0,74^{*}$ & $-3536^{n s}$ & 99 & $22,2 \mathrm{c}$ & $0,96^{\text {ns }}$ & $70,52^{*}$ & 92 \\
\hline URS Tarimba & $2216 \mathrm{~d}$ & $0,83^{*}$ & $52886^{*}$ & 94 & $26,8 \mathrm{c}$ & $0,75^{*}$ & $-14,72^{n s}$ & 99 \\
\hline URS Taura & $1901 \mathrm{e}$ & $0,79 *$ & $43214^{*}$ & 94 & $34,4 \mathrm{~b}$ & $1,30^{*}$ & $153,32^{*}$ & 91 \\
\hline URS 21 & $2480 \mathrm{c}$ & $0,75^{*}$ & $1703^{n s}$ & 98 & $29,2 \mathrm{c}$ & $0,96^{n s}$ & $30,95^{n s}$ & 95 \\
\hline FAEM 007 & $1905 \mathrm{e}$ & $1,19^{*}$ & $85956^{*}$ & 95 & $47,0 \mathrm{a}$ & $1,23^{*}$ & $179,29^{*}$ & 89 \\
\hline FAEM 006 & $2427 \mathrm{c}$ & $1,34^{*}$ & $-5386^{n s}$ & 100 & $43,1 \mathrm{a}$ & $1,55^{*}$ & $24,85^{n s}$ & 98 \\
\hline FAEM 5 Chiarasul & $2327 \mathrm{~d}$ & $1,54^{*}$ & $146478^{*}$ & 96 & $43,4 \mathrm{a}$ & $1,55^{*}$ & $31,38^{n s}$ & 98 \\
\hline FAEM 4 Carlasul & $2611 \mathrm{c}$ & $1,05^{n s}$ & $9304^{n s}$ & 99 & $33,0 \mathrm{~b}$ & $1,07^{\text {ns }}$ & $22,65^{n s}$ & 96 \\
\hline Brisasul & $2630 \mathrm{c}$ & $1,24^{*}$ & $6535^{n s}$ & 99 & $22,5 \mathrm{c}$ & $0,50^{*}$ & $24,00^{n s}$ & 86 \\
\hline Barbarasul & $2463 \mathrm{c}$ & $1,48^{*}$ & $24617^{*}$ & 99 & $46,1 \mathrm{a}$ & $1,47^{*}$ & $506,58^{*}$ & 82 \\
\hline URS Fapa Slava & $2291 \mathrm{~d}$ & $0,99^{n s}$ & $40292^{*}$ & 96 & $33,8 \mathrm{~b}$ & $0,53^{*}$ & $41,53^{*}$ & 83 \\
\hline IPR Afrodite & 2069 e & $1,29 *$ & $-2674^{n s}$ & 100 & $34,8 \mathrm{~b}$ & $1,54^{*}$ & $11,36^{n s}$ & 99 \\
\hline UPFPS Farroupilha & $2613 \mathrm{c}$ & $0,97^{n s}$ & $13982^{\text {ns }}$ & 98 & $23,6 \mathrm{c}$ & $1,01^{\text {ns }}$ & $-21,91^{n s}$ & 100 \\
\hline UPFA Ouro & $2461 \mathrm{c}$ & $0,84^{*}$ & $20431^{*}$ & 97 & $10,6 \mathrm{c}$ & $0,47^{*}$ & $16,52^{n s}$ & 86 \\
\hline UPFA Gaudéria & $2324 \mathrm{~d}$ & $0,8^{*}$ & $27338^{*}$ & 96 & $15,6 \mathrm{c}$ & $0,28^{*}$ & $-18,76^{n s}$ & 95 \\
\hline
\end{tabular}

Médias seguidas pela mesma letra na coluna não diferem significativamente entre si a $5 \%$ de probabilidade de erro; $*$ = significativo a $5 \%$ de probabilidade de erro pelo teste $\mathrm{F} ;{ }^{n s}=$ não significativo; $A F N_{105 D A E}=$ área foliar necrosada aos 105 dias após a emergência; $\mathrm{PG}=$ produtividade de grãos; $b_{0}=$ média geral da cultivar; $b_{1}=$ coeficiente de regressão linear; $S^{2}=$ desvios da regressão; $R^{2}=$ coeficiente de determinação.

\section{Conclusões}

É possível concluir que com o emprego da análise de regressão e do modelo de adaptabilidade e estabilidade de Eberhart \& Russell a cultivar que apresentou melhor desempenho foi a URS Altiva. Desta forma, sua recomendação para cultivo em condições de ano agrícola intermediário à produtividade e favorável à progressão de doenças foliares pode 
promover uma produtividade de grãos satisfatória com a possibilidade de menor uso de fungicida.

\section{Agradecimentos}

Ao CNPq, à CAPES, à FAPERGS e à UNIJUÍ pelos recursos financeiros e estrutura física e material necessários para realização da pesquisa.

\section{Referências}

[1] A. P. Brunes, S. Oliveira, E. S. Lemes, L. C. Tavares, V. M. Gehling, L. W. Dias, F. A. Villela, Adubação boratada e produção de sementes de trigo. Ciência Rural, 45:1572-1578, 2015.

[2] J. A. G. da Silva, M. D. Wohlenberg, E. G. Arenhardt, A. C. Oliveira, G. Mazurkievicz, M. Muller, L. G. Arenhardt, M. O. Binelo, G. Arnold, R. Pretto, Adaptability and stability of yield and industrial grain quality with and without fungicide in Brazilian oat cultivars. American Journal of Plant Sciences, 6:1560-1569, 2015.

[3] J. A. G. da Silva, C. J. G. Neto, S. B. Fernandes, R. D. Mantai, O. B. Scremin, R. Pretto, Nitrogen efficiency in oats on grain yield with stability. Revista Brasileira de Engenharia Agrícola e Ambiental, 20:1095-1100, 2016.

[4] S. A. Eberhart, W. A. Russell, Stability parameters for comparing varieties. Crop science, 6:36-40, 1966.

[5] J. f. Hair, W. C. Black, B. J. Babin, R. E. Anderson, R. L. Tatham, Análise Multivariada de Dados. Bookman, Porto Alegre, 2005.

[6] A. Marolli, J. A. G. da Silva, M. V. Romitti, R. D. Mantai, O. B. Scremin, R. Z. Frantz, S. Sawicki, E. G. Arenhardrt, M. E. Gzergorczick, A. R. C. Lima, Contributive effect of growth regulator Trinexapac-Ethyl to oats yield in Brazil, African Journal of Agricultural Research, 12:795-804, 2017. DOI:10.5897/AJAR2016.11784.

[7] J. M. J. Nerbass, R. T. Casa, P. R. J. Kuhnem, F. Gava, A. Bogo, Modelos de pontos críticos para relacionar o rendimento de grãos de aveia branca com a intensidade de doença no patossistema múltiplo ferrugem da folha: helmintosporiose. Ciência Rural, 40:1-6, 2010.

[8] M. V. Romitti, J. A. G. da Silva, A. Marolli, E. G. Arenhardr, A. T. W. de Mamann, O. B. Scremin, O. A. Lucchese, C. A. M. B. Kruger, L. G. Arenhardr, L. M. Bandeira, The management of sowing density on yield and lodging in the main oat biotype grown in Brazil. African Journal of Agricultural Research, 11:1935-1944, 2016.

[9] A. J. Scott, M. A. Knott, Cluster analysis method for grouping means in the analysis of varience. Biometric, 30:507-512, 1974. 